\title{
Modular control of gait after incomplete spinal cord injury: differences between sides
}

\author{
S Pérez-Nombela ${ }^{1,6}$, F Barroso ${ }^{2,3,6}$, D Torricelli², A de los Reyes-Guzmán ${ }^{1}$, AJ del-Ama ${ }^{1}$, J Gómez-Soriano ${ }^{4,5}$, \\ JL Pons ${ }^{2}$ and Á Gil-Agudo ${ }^{1}$
}

Study design: This is an analytical descriptive study.

Objectives: The main goal of this study was to compare the modular organization of bilateral lower limb control in incomplete spinal cord injury (iSCl) patients during overground walking, using muscle synergies analysis. The secondary goal was to determine whether the similarity between the patients and control group correlate with clinical indicators of walking performance.

Setting: This study was conducted in National Hospital for Spinal Cord Injury (Toledo, Spain).

Methods: Eight iSCl patients and eight healthy subjects completed 10 walking trials at matched speed. For each trial, threedimensional motion analysis and surface electromyography (sEMG) analysis of seven leg muscles from both limbs were performed. Muscle synergies were extracted from sEMG signals using a non-negative matrix factorization algorithm. The optimal number of synergies has been defined as the minimum number needed to obtain variability accounted for (VAF) $\geqslant 90 \%$.

Results: When compared with healthy references, iSCI patients showed fewer muscle synergies in the most affected side and, in both sides, significant differences in the composition of synergy 2 . The degree of similarity of these variables with the healthy reference, together with the composition of synergy 3 of the most affected side, presented significant correlations $(P<0.05)$ with walking performance.

Conclusion: The analysis of muscle synergies shows potential to detect differences between the two sides in patients with iSCl. Specifically, the VAF may constitute a new neurophysiological metric to assess and monitor patients' condition throughout the gait recovery process.

Spinal Cord (2017) 55, 79-86; doi:10.1038/sc.2016.99; published online 28 June 2016

\section{INTRODUCTION}

A damage to the spinal cord results in a partial or complete loss of motor, sensory and autonomic functions below the level of the lesion. ${ }^{1}$ In particular, the interruption of the spinal interneuronal circuitry connecting the brainstem and the supraspinal motor center interferes in the quality of gait. ${ }^{2}$ Improving gait function after spinal cord injury (SCI) is one of the most important milestones in the rehabilitation process, as well as one of the factors with the greatest impact on the social and professional reintegration of the patient. ${ }^{3}$

A main challenge in gait training is the progressive adaptation to the patient-specific reorganization of sensorimotor functions. ${ }^{4}$ To this aim, the accurate measurement of locomotor responses after neurological disease is perceived as a central issue to achieve this goal. ${ }^{5}$ The kinematic measures derived from gait analysis provide a detailed and quantitative description of the motor behavior of patients with gait disturbances. For instance, in previous biomechanical studies, we have observed remarkable differences localized at the knee and ankle levels, in the sagittal plane, between incomplete spinal cord injury (iSCI) patients and healthy subjects. ${ }^{6,7}$ Thus, the information provided by kinematic measures may be of great value to help the physician to set a specific rehabilitation program.
In addition to kinematic analysis, surface electromyography (sEMG) may provide valuable information about disturbances and changes in motor control in neurological diseases, and therefore it is used to guide the rehabilitation process. ${ }^{4}$ In particular, several recent studies, in humans ${ }^{8-13}$ and simulations, ${ }^{14,15}$ support the hypothesis that walking functions result from synergistic control of muscles. Muscle synergies, also known as motor modules, can be defined as functional units of the nervous system that coordinate the coactivation of different groups of muscles. ${ }^{16}$ Recent studies on the modular control of gait have shown that the activation of leg muscles during human locomotion can be reconstructed as a linear combination of four to five muscle synergies, each of which are activated at a different phase of the gait cycle ${ }^{17-19}$ and underlying a specific biomechanical function. ${ }^{15,20,21}$

Neurological impairments may alter the normal functioning of modular control and lead to gait disturbances. ${ }^{16}$ In the case of poststroke patients, the presence of a fewer number of synergies has been observed in the affected side. ${ }^{18,22,23}$ This reduction has been associated with an overall decrease in the complexity of locomotor control, expressed by a lower number of independently activated groups of muscles. In line with these findings, Bowden et al..$^{23}$ referred

${ }^{1}$ Biomechanical and Technical Aids Department, National Hospital for Spinal Cord Injury, Toledo, Spain; ${ }^{2}$ Neural Rehabilitation Group, Cajal Institute, Spanish National Research Council (CSIC), Madrid, Spain; ${ }^{3}$ Centre ALGORITMI, University of Minho, Azurém, Guimarães, Portugal; ${ }^{4}$ Sensoriomotor Function Group, National Hospital for Spinal Cord Injury, Toledo, Spain and ${ }^{5}$ Toledo Physiotherapy Research Group (GIFTO). Nursing and Physical Therapy School, Castilla-La Mancha, Toledo, Spain

${ }^{6}$ These authors contributed equally to this work.

Correspondence: Dr S Pérez-Nombela, Biomechanical and Technical Aids Department, National Hospital for Spinal Cord Injury, Finca La Peraleda s/n, Toledo 45071, Spain. E-mail: sperezn@sescam.jccm.es

Received 9 October 2015; revised 21 March 2016; accepted 24 May 2016; published online 28 June 2016 
to the number of muscle synergies as a potential predictor of walking performance, especially in the case of poor intermuscular coordination. Thus, the analysis of muscle synergies might be explored as a quantitative indicator of the changes in muscle activation patterns and coordination in pathological gait. ${ }^{24}$

In the case of SCI, the few existing reports suggest that muscle synergies are also affected after the injury. However, because of the high heterogeneity of location and magnitude of the lesion, common features in the modular control across SCI patients are hardly reported. ${ }^{25}$ Fox et al. ${ }^{16}$ found that less muscle synergies were needed to account for most of the variability of muscle activation in the lower limbs of children with iSCI in different motor tasks in comparison with healthy children. However, these findings must be analyzed with caution, as the modular control in children may differ substantially from adults, as their nervous system is still immature and developing. ${ }^{16}$ Other authors studied heterogeneous populations, including patients with complete motor SCI, where they analyzed different conditions of weight loading and speed. ${ }^{19,26,27}$ In these studies, patients were constrained to treadmill walking, which might include significant differences when compared with overground walking. ${ }^{28}$ Only Hayes et al. ${ }^{29}$ have conducted a study to quantify neuromuscular deficits in muscle coordination in iSCI subjects during overground gait, concluding that the number of muscle synergies, as well as their composition and activations, were significantly altered in these patients. ${ }^{29}$ The authors also highlighted that one limitation of their study was that EMG was measured only from one side of the body, whereas iSCI may affect both sides differently, leading to an asymmetric motor control and functional behavior.

On the basis of these previous findings, the main goal of this study was to analyze the bilateral differences in synergistic control in iSCI patients. The secondary goal was to find a correlation between the healthy-like modular organization of muscle synergies in iSCI patients and indicators of walking performance, assessed with functional clinical scales. Our main hypothesis is that patients' muscle synergies present significant differences between sides, when a functional asymmetry is present. Our secondary hypothesis is that the degree of similarity of the muscle synergies with a healthy data set will correlate with functional clinical scales as indicators of motor performance. The confirmation of these two hypotheses would support the importance of studying the bilateral control of lower limb functions in iSCI patients, as well as support the use of muscle synergies as a metric of neuromotor performance and as a complementary tool to clinical scales and kinematic measures.

\section{MATERIALS AND METHODS}

\section{Subjects}

Eight iSCI patients ( 5 men and 3 women; $38.38 \pm 10.45$ year) participated in the experiment. Individual information of the patients is given in Table 1. Inclusion criteria were as follows: age between 18 and 65 years, motor incomplete spinal lesion (ASIA Impairment Scale, AIS C-D), evolution of at least 1.5 months, the absence of any other pathological condition and the ability to walk $10 \mathrm{~m}$ unassisted with or without technical aids. Exclusion criteria were history of epilepsy, passive restriction of the joints and diagnosis of any other disease associated with memory, concentration and/or visual deficits. Eight healthy subjects ( 4 men and 4 women; $31.50 \pm 6.61$ year) with similar demographic and anthropometric characteristics, and with no diagnosed neural injury, neither central nor peripheral, served as a control group (CG).

All applicable institutional and governmental regulations concerning the ethical use of human volunteers were followed during the course of this research, and all recruited participants provided written consent before the beginning of the experiments. The local Ethical Committee approved the experimental protocol design. The research was carried out in the Biomechanical and Technical Aids Department of the National Hospital for Spinal Cord Injury (Toledo, Spain).

\section{Experimental protocol and data collection}

Before the experiment, a trained physiotherapist performed a set of clinical evaluations to assess the clinical and functional status of the patients. Their clinical characteristics and functional gait performance were evaluated using the following scales: lower extremity motor score (LEMS), which analyzes five key muscles in each leg, with a cumulative score for each extremity being between 0 and 25 and a total score from 0 and 50; the walking index for spinal cord injury II (WISCI II), which assesses the level of walking depending on the use of devices, braces and physical assistance to walk $10 \mathrm{~m}$, on a scale from 0 to 20 ; $10-\mathrm{m}$ walking test (10MWT), which measures the time spent to walk $10 \mathrm{~m}$ at self-selected speed and using the usual walking devices; timed up and go (TUG), which measures the time spent to stand up from a chair, walking $3 \mathrm{~m}$, turn around, return to the chair and sit down again at self-selected speed and using the habitual walking devices; and the spinal cord independence measure (SCIM), which is a disability scale for $\mathrm{SCI}^{3,7,30}$ (Table 1).

Gait analysis was carried out using a three-dimensional motion analysis system with two scanner units (CODA System6, Charnwood Dynamics, Ltd, Leicester, UK). Eleven active markers were positioned and attached to anatomic landmarks, as described previously. ${ }^{31}$ Data were recorded with an acquisition frequency of $200 \mathrm{~Hz}$. Each participant completed 10 walking trials along a 10- $\mathrm{m}$ walkway at self-selected speed, resting one minute between trials to avoid fatigue. All the iSCI patients walked with their usual footwear. CG participants were asked to walk within the range of speeds of iSCI group, following the same criterion of Ochi et al. ${ }^{32}$ and Gil-Agudo et al. ${ }^{7}$

For all iSCI patients, surface electromyography (EMG) data were recorded bilaterally using an EMG recording system (Noraxon, Scottsdale, AZ, USA), with an acquisition frequency of $1500 \mathrm{~Hz}$, synchronized with the CODA system. In the case of CG, only the right limb was evaluated. Surface EMG

Table 1 Individual patients' information

\begin{tabular}{|c|c|c|c|c|c|c|c|c|c|c|c|c|c|}
\hline $\begin{array}{l}\text { Subject } \\
I D\end{array}$ & Gender & $\begin{array}{c}\text { Age } \\
\text { (years) }\end{array}$ & $\begin{array}{l}\text { Level of } \\
\text { lesion }\end{array}$ & AIS & $\begin{array}{c}\text { Months after } \\
\text { injury }\end{array}$ & $\begin{array}{c}\text { WISCI } \\
\quad / I\end{array}$ & Assistive device & $\begin{array}{c}\text { LEMS }(L / R \\
\text { total) }\end{array}$ & $10 M W T$ & TUG & SCIM & $\begin{array}{c}\text { Cadence in steps/minute } \\
(L / R)\end{array}$ & $\begin{array}{c}\text { Most affected } \\
\text { side }\end{array}$ \\
\hline iSCl1 & $M$ & 25 & $\mathrm{C} 4$ & $\mathrm{D}$ & 4 & 19 & One crutch (right) & $16 / 21,37$ & 27 & 32 & 68 & $42.3 / 53.5$ & Left \\
\hline iSCl2 & M & 36 & $\mathrm{C7}$ & D & 154 & 20 & None & $21 / 25,46$ & 12.5 & 10 & 93 & $73.8 / 76.5$ & Left \\
\hline iSCl3 & M & 51 & $\mathrm{~T} 12$ & D & 216 & 20 & None & $21 / 20,41$ & 8 & 8 & 93 & $103.4 / 101.8$ & Right \\
\hline iSCl4 & M & 41 & C6 & D & 6 & 20 & None & $24 / 24,48$ & 9 & 10 & 83 & $95 / 86.7$ & Right \\
\hline iSCI5 & M & 31 & C6 & $\mathrm{D}$ & 3 & 19 & One crutch (right) & $18 / 24,42$ & 10 & 11 & 83 & $85.7 / 91.8$ & Left \\
\hline iscl6 & $\mathrm{F}$ & 48 & $\mathrm{C5}$ & D & 3 & 20 & None & $24 / 25,49$ & 7 & 6.15 & 99 & $101 / 99.2$ & Right \\
\hline iSCl7 & $\mathrm{F}$ & 26 & $\mathrm{~T} 10$ & D & 6 & 19 & One crutch (right) & $22 / 22,44$ & 8 & 9 & 83 & $84.4 / 79.5$ & Right \\
\hline iSCl8 & $\mathrm{F}$ & 49 & $\mathrm{T7}$ & $\mathrm{D}$ & 7 & 12 & $\begin{array}{l}\text { Two crutches and } \\
\text { braces }\end{array}$ & $17 / 22,39$ & 18 & 21 & 85 & $68.9 / 74.8$ & Left \\
\hline
\end{tabular}

Abbreviations: AIS, ASIA Impairment Scale; WISCI II, Walking index for spinal cord injury; D, preservation of motor function with $50 \%$ or more of key muscles below the neurological level, having a muscle grade of $\geqslant 3 ; \mathrm{F}$, female; L, left; LEMS, lower extremity motor score; M, male; R, right; SCIM, spinal cord independence measure; TUG, timed up and go; 10MWT, 10-m walking test. 
electrodes were positioned as described in Cram et al. ${ }^{33}$ on the following seven muscles: gluteus medius (GlMe), gluteus maximus (GlMa), rectus femoris (ReFe), adductor longus (AdLl), medial hamstrings (Ham), tibialis anterior (TiAn) and gastrocnemius medialis (Gas).

EMG and CODA data were analyzed offline using MATLAB R2011a (The MathWorks, Natick, MA, USA) and SPSS 17 (IBM SPSS Inc., Chicago, IL, USA).

\section{Data analysis}

For each trial, a single gait cycle corresponding to the midpoint of the $10-\mathrm{m}$ walkway was selected, to ensure that the gait pattern was not influenced by initial acceleration and final braking. For each of the 10 trials performed by each subject, one left stride and one right stride were selected for analysis. Each walking stride started at heel strike of the corresponding leg, determined by visual inspection from the heel marker trajectory. Cadence and speed were calculated independently for each side, according to the time spent and distance walked during a step. Cadence values of each side were used to tag sides as most or less affected, with the most affected side (iSCIa) being the one achieving a lower cadence (Table 1). Finally, data from the 10 gait cycles were concatenated according to the side recorded.

Concatenated raw EMG signals were pre-processed using a high-pass filter with cutoff frequency of $20 \mathrm{~Hz}$, demeaned, rectified and smoothed with a lowpass filter with a cutoff frequency of $5 \mathrm{~Hz}$, resulting in the EMG envelopes. ${ }^{8,18}$ EMG envelopes from each muscle were normalized by the average of the maximum values of each of the 10 strides, and then resampled in time at $1 \%$ of the stride cycle. For each subject and analyzed side, the normalized EMG envelopes were combined into an $m \times t$ matrix $\left(\mathrm{EMG}_{0}\right)$, where $m$ is the number of muscles (seven in this case) and $t$ is the time base ( $t=$ number of cycles $(10) \times 100)$ ). Muscle synergies were extracted using the non-negative matrix factorization (NNMF) algorithm. ${ }^{34}$ Mathematically, the output of the algorithm is described in the following equation:

$$
\mathrm{EMG}_{0}=\mathrm{WH}+e=\mathrm{EMG}_{\mathrm{r}}+e
$$

where $W$ is a $m \times n$ matrix ( $n$ is the number of muscle synergies) that specifies the relative weighting of each muscle within each synergy (hereafter, each column of $W$ will be referred to as muscle synergy vector); $H$ is a $n \times t$ matrix specifying the time-varying activation coefficients, which represent the recruitment of each synergy vector over time; $\mathrm{EMG}_{\mathrm{r}}$ is an $m \times t$ matrix resulting from the multiplication of $W$ and $H$, representing the reconstructed EMG envelopes; and $e$ is the residual error. For each $\mathrm{EMG}_{0}$, the NNMF algorithm was run six times, each time considering a different number of synergies, from 2 to 7 ( $n=2$, $3,4,5,6,7)$. To avoid local minima, for each run the NNMF was repeated 40 times and the repetition with the lowest reconstruction error was selected.

The number of muscle synergies is usually determined based on a reconstruction quality criterion. ${ }^{18}$ We decided to use the variability accounted for $\left(\mathrm{VAF}_{\text {total }}\right)^{8,18}$ to evaluate the quality of reconstruction of the EMG data, according to the following equation:

$$
\operatorname{VAF}_{\text {total }}=1-\frac{\sum_{i=1}^{m} \sum_{j=1}^{t}\left(\mathrm{EMG}_{0}(i, j)-\mathrm{EMG}_{r}(i, j)\right)^{2}}{\sum_{i=1}^{m} \sum_{j=1}^{t}\left(\mathrm{EMG}_{0}(i, j)\right)^{2}}
$$

For each data set processed, the criterion used to estimate the number of synergies was defined as the minimum number needed to obtain $\mathrm{VAF}_{\text {total }}$ values $\geqslant 90 \%{ }^{35}$

Data from healthy subjects (CG) were used to obtain two reference matrices (hereafter called $W_{0}$ and $H_{0}$ ) by pooling the EMG envelopes from the CG, and by applying the NNMF algorithm. In this case, the number of synergies was set to four, regardless of the $\mathrm{VAF}_{\text {total }}$ criteria, as done previously by Clark et al. ${ }^{18}$ and Routson et al..$^{35}$ This allowed us to compare muscle synergy composition and activation of iSCI patients with the four healthy synergies described in literature for healthy subjects. ${ }^{13,18}$ The similarity between patients and healthy subjects was computed by, first, ordering muscle synergy vectors (columns of matrix $W$ ) of each patient according to their similarity with columns of $W_{0}$, and then correlating synergy vectors $(W)$ and activation coefficients $(H)$ with the corresponding vectors from the healthy data set $\left(W_{0}\right.$ and $\left.H_{0}\right)$. Similarity and correlation values were computed using the normalized scalar product. ${ }^{8}$ Hereafter, the normalized scalar products between $H$ and $H_{0}$ and between $W$ and $W_{0}$ are denoted ' $H \cdot H_{0}$ ' and ' $W \cdot W_{0}$ ', respectively.

\section{Statistical analysis}

A descriptive analysis of the clinical and functional variables was obtained by calculating the mean and s.d. for the quantitative variables, and the frequencies and percentages for the qualitative variables. Samples were analyzed with Kolmogorov-Smirnov test.

After verifying the normality of the samples, we used one-way ANOVA with post hoc Bonferroni for multiple comparisons between the three groups (control group, most affected side of iSCI patients and less affected side of iSCI patients) in terms of speed, cadence, $\mathrm{VAF}_{\text {total }}$ and the number of synergies. To detect differences between both sides of patients with iSCI, we performed paired $t$-tests. Finally, Pearson's correlation was used to test for significant relationships between functional clinical scales and $\mathrm{H} \cdot \mathrm{H}_{0}$ and $\mathrm{W} \cdot \mathrm{W}_{0}$.

Statistical significance was set by a $P$-value of 0.05 .

\section{RESULTS}

\section{Speed and cadence}

Patients' speed was very heterogeneous, ranging from 0.30 to $1.05 \mathrm{~m} \mathrm{~s}^{-1}$ in both sides, with an average speed of $0.73 \pm 0.25 \mathrm{~m} \mathrm{~s}^{-1}$ (mean \pm s.d.) in the less affected side and $0.71 \pm 0.24 \mathrm{~m} \mathrm{~s}^{-1}$ in the most affected side. The CG walked at an average speed of $0.92 \pm 0.20 \mathrm{~m} \mathrm{~s}^{-1}$. Differences in speed were not statistically significant when comparing the speed of the three groups $(P>0.05$; Table 2$)$.

Regarding iSCI patients' cadence, values ranged globally from 42.28 to 103.40 steps per min. Considering the two legs separately, cadence was $85.05 \pm 16.52$ steps per min for the less affected side and $79.75 \pm 18.92$ steps per min for the most affected side. CG walked with a cadence of $87.83 \pm 7.60$ steps per min. No statistically significant differences were found among the analyzed groups $(P>0.05$; Table 2)

\section{Muscle synergies organization}

The most affected side of iSCI patients showed a fewer number of muscle synergies $(2.88 \pm 0.64)$ compared with both the less affected side $(3.38 \pm 0.52)$ and the CG $(3.62 \pm 0.52)$. In the latter case, the differences were statistically significant. This finding indicates that the most affected side of iSCI patients presents less complexity in terms of independent activation while walking overground (Table 3).

Considering the most affected side, two patients (25\%) showed two synergies, five patients (62.5\%) required three synergies and one

Table 2 Differences between the three analyzed groups, when comparing speed and cadence

\begin{tabular}{lcccc}
\hline Variable (units) & iSCl & iSCla & CG & P-value \\
\hline Speed $\left(\mathrm{m} \mathrm{s}^{-1}\right.$ ) & $0.73 \pm 0.25$ & $0.71 \pm 0.24$ & $0.92 \pm 0.20$ & 0.158 \\
Cadence (steps per min) & $85.05 \pm 16.52$ & $79.75 \pm 18.92$ & $87.83 \pm 7.60$ & 0.565
\end{tabular}

Values are expressed in mean \pm s.d. iSCl, less affected side of each iSCl patient; iSCla, most affected side of each iSCl patient; CG, right side from each subject belonging to the control group.

Table 3 Number of muscle synergies and VAF $_{\text {total }}$ values obtained for the three groups, when using two to four synergies to reconstruct overall muscle activity

\begin{tabular}{lcccc}
\hline Variable & iSCl & iSCla & CG & P-value \\
\hline Number of synergies & $3.38 \pm 0.52$ & $\mathbf{2 . 8 8} \pm \mathbf{0 . 6 4}$ & $\mathbf{3 . 6 2} \pm \mathbf{0 . 5 2}$ & $\mathbf{0 . 0 4 2}$ \\
VAF total (two synergies) & $83.44 \pm 4.60$ & $85.20 \pm 5.40$ & $81.80 \pm 2.75$ & 0.321 \\
VAF $_{\text {total }}$ (three synergies) & $90.25 \pm 2.58$ & $91.88 \pm 1.66$ & $90.39 \pm 2.12$ & 0.269 \\
VAF $_{\text {total }}$ (four synergies) & $94.51 \pm 1.35$ & $95.48 \pm 1.00$ & $94.83 \pm 1.49$ & 0.332
\end{tabular}

Values are expressed as mean \pm s.d. $P$-value is from one-way ANOVA. Bold values indicate significant differences after applying post hoc Bonferroni. 


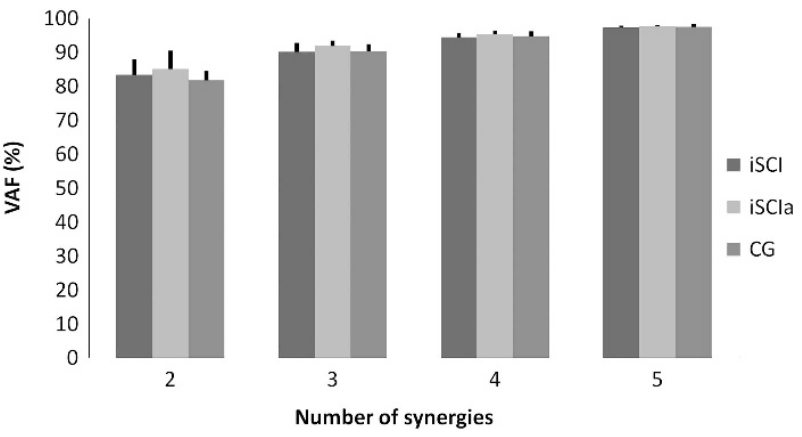

Figure 1 Total variability accounted for $\left(\mathrm{VAF}_{\text {total }}\right)$ values based on the number of synergies used as input to the NNMF algorithm to reconstruct EMG activity. The bars represent the mean of $\mathrm{VAF}_{\text {total }}$ for each number of synergies per group. Each bar has an error bar based on one s.d.

patient (12.5\%) showed four synergies. In the case of the less affected sides, five patients $(62.5 \%)$ required three synergies, and the remaining three patients $(37.5 \%)$ required four synergies. In the CG, three subjects $(37.5 \%)$ required three synergies, and the remaining five $(62.5 \%)$ needed four synergies (Figure 1).

Similar to what was previously performed by Clark et al. ${ }^{18}$ and Routson et al. ${ }^{35}$ muscle synergy vectors $(W)$ and activation coefficients $(H)$ were extracted assuming the existence of four muscle synergies, regardless of the $\mathrm{VAF}_{\text {total }}$ criterion, in order to allow for comparison across subjects. ${ }^{18,35}$

The four muscle synergies extracted with concatenated data from the control group were consistent with those described in literature

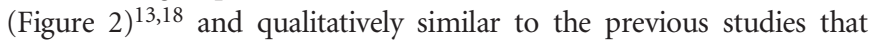
recorded a larger set of muscles. ${ }^{20,21}$ Synergy 1 mainly involved the activity of hamstrings (knee flexors and hip extensors) at the end of swing and initial heel contact. Synergy 2 described the activity of GIMe (hip abductor) and GIMa (hip extensor and abductor), ReFe (knee extensor and hip flexor) and TiAn (ankle dorsiflexor) during early stance. Synergy 3 mainly involved the activity of Gas (ankle plantarflexor) and, to a lower extent, GIMa, during late stance. Finally, synergy 4 consisted in the activation of AdLo (hip adductor), TiAn and, to a lower extent, ReFe during initial swing phase.

Both muscle synergy vectors $(W)$ and activation coefficients $(H)$ were very heterogeneous in both sides of iSCI patients. When comparing the similarity of $H$ with the reference $H_{0}$, we found a significant difference between sides in the $H 2 \cdot H 2_{0}$ index $(P=0.046$; Table 4). Specifically, the activation coefficient of synergy 2 from the less affected side was more similar to the healthy reference than that of the most affected side (Figure 3).

\section{Correlations with clinical scales}

There were no significant correlations between the number of synergies calculated for each side and functional assessment scales. In addition, no significant correlations were found between $\mathrm{VAF}_{\text {total }}$ and functional assessment scales (Table 5).

Significant correlations were found between functional assessment scales and $H 2 \cdot H 2_{0}$ and $W 3 \cdot W 3_{0}$ of the most affected side, when using four synergies to reconstruct the EMG signals. These two indicators presented significant correlations $(P<0.05)$ with $10 \mathrm{MWT}$, TUG and cadence, as shown in Figure 4. In the case of 10MWT and TUG tests, correlations were negative, meaning that the more time patients needed to perform these tests the less similar $W 3$ and $H 2$ were with the healthy reference. In the case of cadence, the correlation was positive: patients walking at higher cadence presented higher similarity to the reference from CG in what concerns to $W 3$ and $H 2$.

\section{DISCUSSION}

The main purpose of this study was to assess the bilateral modular organization of lower limbs in iSCI patients during overground walking, under the hypothesis that gait patterns after an SCI are not always symmetrical between limbs.

Because of the influence of walking speed on the number of muscle synergies, ${ }^{18}$ we asked the CG to walk within the range of speeds of iSCI group, to allow for a more truthful comparison across groups. Moreover, the trials were performed overground, because comfortable speed may differ in populations with motor impairments if walking on a treadmill. ${ }^{23}$

In clinical practice, the most affected side is usually determined by LEMS score. ${ }^{7,36}$ As four out of the eight iSCI patients presented equal or very similar LEMS score between sides, we added an additional criterion based on the cadence achieved by each side independently, according to the time spent to perform a step, with the most affected side being the one that needed more time. This additional criterion seems to be robust and useful to determine the most affected side in iSCI patients, as further differences were found between sides.

Results showed that fewer muscle synergies were needed in the most affected side when compared with the less affected side. The identification of less muscle synergies is associated with lower independence among the activation patterns of each muscle, possibly unveiling a coupling mechanism across muscles or a merging of existing muscle synergies. ${ }^{9}$ Similar results have been reported when comparing the paretic and nonparetic sides of poststroke patients. ${ }^{18}$

There is evidence in the literature that SCI patients present less muscle synergies than healthy controls, ${ }^{16,29}$ despite these reports being analyzed only the right limb of patients. In our study, we obtained the same evidence when analyzing the most affected side of iSCI patients.

We found globally up to four synergies in all groups analyzed. Each of these four muscle synergies has been associated, in healthy individuals, with a specific biomechanical function. ${ }^{15,20,21}$ In this analysis, we found that the synergy 1 consisted mainly of Ham activation (hip extensor and knee flexor), which were activated in the terminal swing phase and initial heel contact to decelerate the lower limb during the final gait cycle and to prepare for the initial contact. ${ }^{15,17,18}$ The second synergy consisted mainly of the activation of GIMe (hip abductor), GIMa (extensor and abductor hip) and ReFe (hip flexor and knee extensor), which were active during the loading response phase to support the body and to accept the body weight during initial contact; ${ }^{15,17,18}$ TiAn (ankle dorsiflexor) was also activated by synergy 2, probably to ensure contact with the heel and not with the forefoot. Synergy 3 consisted mainly of Gas (ankle plantarflexor) activation during mid and terminal stance phase, contributing to body support and forward propulsion; ${ }^{15,17,18}$ GIMe was also activated by this synergy, which might prevent pelvic drop during the monopodal stance and the Trendelenburg gait pattern. Synergy 4 was mainly composed by the activation of TiAn and ReFe during initial swing phase, contributing to foot clearance; ${ }^{15,17,18}$ AdLl (hip adductor) was also activated by this synergy, providing stability in the frontal plane of hip during the terminal stance. ${ }^{29}$

When observing the synergistic control in the iSCI group, our results resemble the findings obtained by Ivanenko et al. ${ }^{19}$ We found that the composition of synergies depended on the severity of the injury, presenting higher similarity with the healthy reference in patients with iSCI classified as AIS C or D. ${ }^{19}$ Conversely, in the most affected subjects, the activation coefficients of synergy $2(H 2)$ were 
a
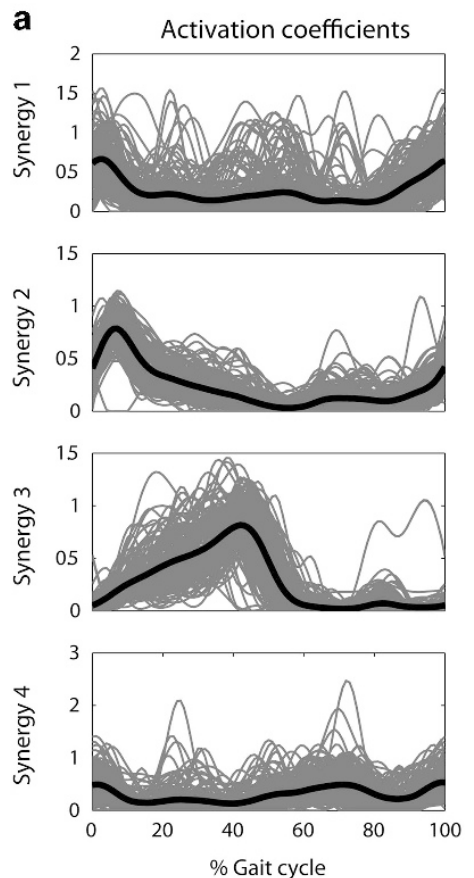

b

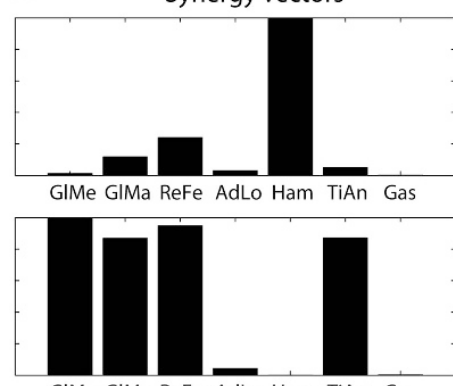

GIMe GIMa ReFe AdLo Ham TiAn Gas

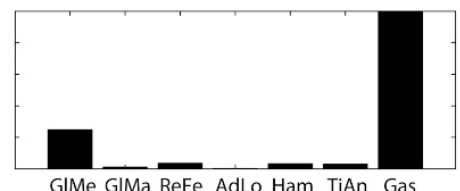

GIMe GIMa ReFe AdLo Ham TiAn Gas

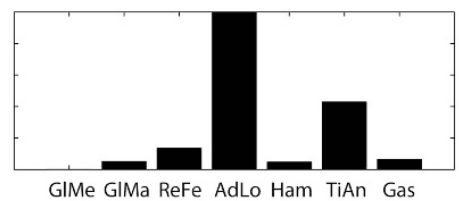

GIMe GIMa ReFe AdLo Ham TiAn Gas
C Original and reconstructed EMG
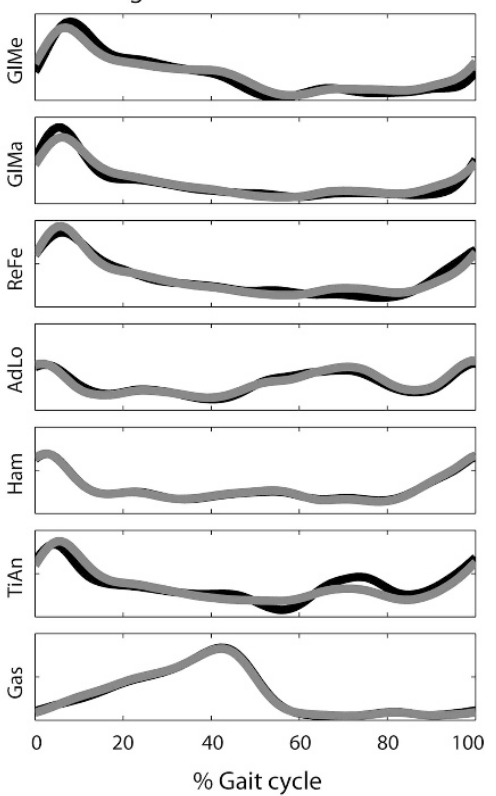

Figure 2 Reconstructed EMGs from the eight healthy subjects from the CG with the NNMF algorithm, using four muscle synergies. (a) Activation coefficients (rows of matrix $H_{0}$ ) of the reference set indicate time-varying profiles of activation responsible to activate each synergy. Thin gray lines represent activation coefficients of each of the 80 strides (10 stride cycles/healthy subject), with each black thick line representing the average of those cycles. (b) Synergy vectors (columns of matrix $W_{0}$ ) of the reference set indicate the relative weighting of each synergy for each muscular pattern. (c) Average of original EMG envelopes from matrix $\mathrm{EMG}_{0}$ (black lines) and reconstructed $\mathrm{EMG}$ patterns from matrix $\mathrm{EMG}_{r}$ (gray lines), resulting from the multiplication of $W 0$ and $H O$.

Table 4 Similarity of synergy vectors $\left(W \cdot W_{0}\right)$ and activation coefficients $\left(H \cdot H_{0}\right)$ with the CG reference, using four synergies to reconstruct the EMG envelopes of the less affected side (iSCl) and the most affected side (iSCla) of iSCI patients

\begin{tabular}{|c|c|c|c|c|c|c|c|c|}
\hline Subject ID & $W 1 \cdot W 1_{0}$ & $W 2 \cdot W 2_{0}$ & w3. W3o & $W 4 \cdot W 4_{0}$ & $H 1 \cdot H 1_{O}$ & $\mathrm{H} 2 \cdot \mathrm{H}_{2} \mathrm{O}$ & $H 3 \cdot H 3_{O}$ & $\mathrm{H} 4 \cdot \mathrm{H}_{0} \mathrm{O}$ \\
\hline iscl2 & 0.88 & 0.92 & 0.81 & 0.84 & 0.73 & 0.91 & 0.96 & 0.89 \\
\hline iSCl4 & 0.15 & 0.76 & 1.00 & 0.90 & 0.76 & 0.95 & 0.94 & 0.94 \\
\hline iSCI5 & 0.87 & 0.77 & 0.94 & 0.55 & 0.85 & 0.93 & 0.89 & 0.80 \\
\hline iscl6 & 0.91 & 0.99 & 0.96 & 0.98 & 0.88 & 0.92 & 0.99 & 0.78 \\
\hline mean \pm s.d. iSCl & $0.57 \pm 0.38$ & $0.81 \pm 0.11$ & $0.93 \pm 0.08$ & $0.83 \pm 0.15$ & $0.82 \pm 0.06$ & $0.87 \pm 0.12$ & $0.86 \pm 0.16$ & $0.83 \pm 0.11$ \\
\hline iSCla1 & 0.98 & 0.80 & 0.71 & 0.65 & 0.82 & 0.54 & 0.93 & 0.75 \\
\hline iscla2 & 0.88 & 0.80 & 0.80 & 0.66 & 0.73 & 0.78 & 0.87 & 0.86 \\
\hline iscla3 & 0.76 & 0.83 & 0.93 & 0.47 & 0.94 & 0.85 & 0.53 & 0.94 \\
\hline isCla4 & 0.91 & 0.91 & 0.96 & 0.83 & 0.93 & 0.93 & 0.94 & 0.90 \\
\hline iscla5 & 0.79 & 0.70 & 0.86 & 0.88 & 0.90 & 0.71 & 0.94 & 0.43 \\
\hline$P$-value & 0.089 & 0.949 & 0.070 & 0.321 & 0.370 & 0.046 & 0.816 & 0.561 \\
\hline
\end{tabular}

Values close to 1 mean high similarity with the healthy reference. Similarities lower than 0.75 appear in bold. Bold values in the last row indicate significant differences between sides.

activated later in the gait cycle when compared with the normal reference $\left(H_{0}\right) . H 2$ is mainly responsible for the activation of ReFe, GIMe and GIMa, all of which are needed for body weight support. As a consequence of the later activation, these patients walk slower to ensure the body weight support and allow the contralateral limb to enter the swing phase. This justifies the significant correlation between functional assessment scales and $\mathrm{H} 2 \cdot \mathrm{H}_{0}$, as patients with more abnormal values of $\mathrm{H} 2$ activation $\left(H 2 \cdot H 2_{0}\right)$ needed more time to perform functional gait tests. Similarly, also synergy vector 3 (W3) shows low similarity with the healthy reference $\left(W 3_{0}\right)$, resulting in a correlation of the $W 3 \cdot W 3_{0}$ index with functional assessment scales. Synergy 3 was mainly responsible for the activation of Gas, necessary 

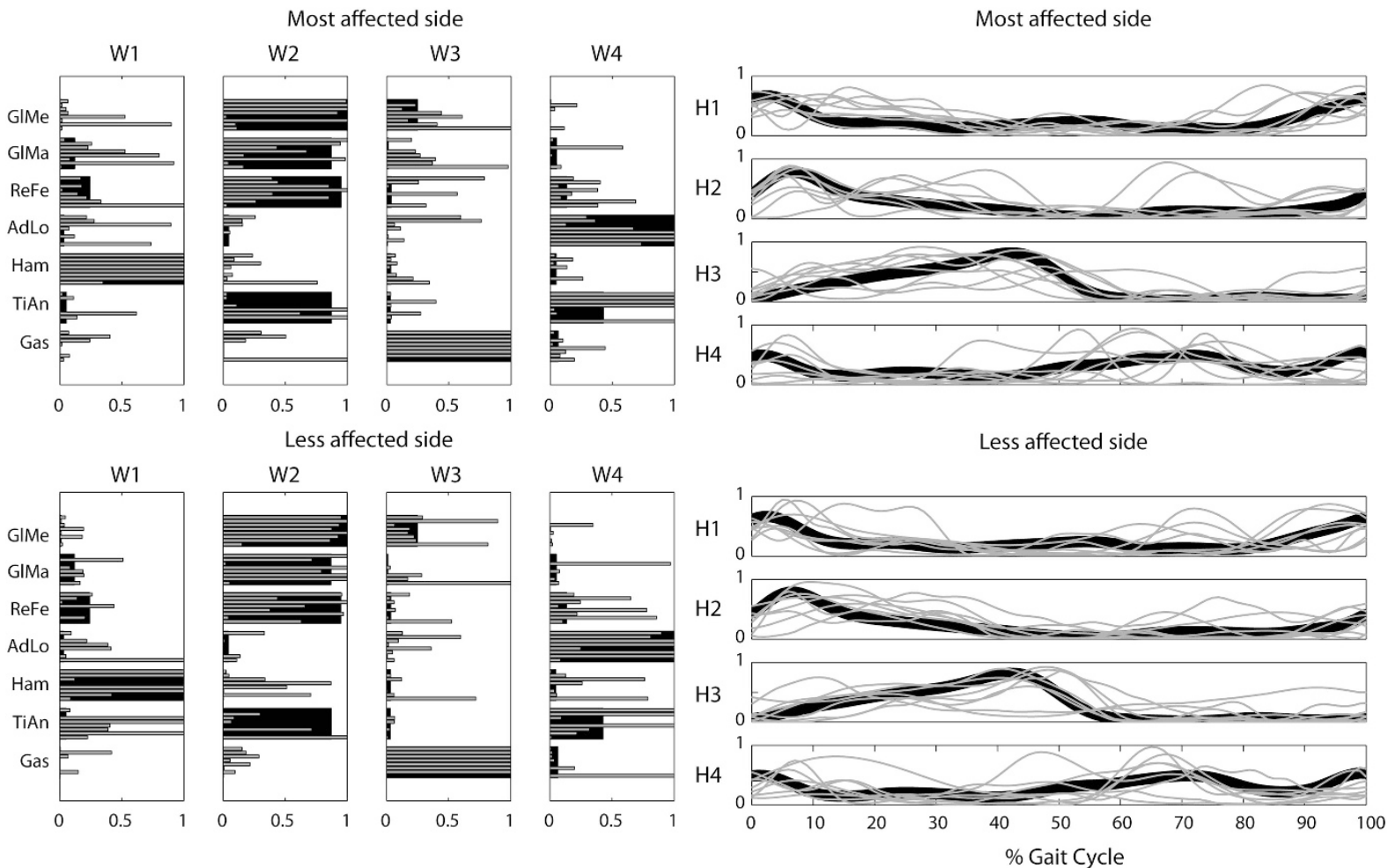

Figure 3 Upper plot: synergy vectors $(W$ ) and activation coefficients $(H)$ extracted for the most affected side of iSCl patients, considering four synergies. Low plot: synergy vectors $(W)$ and activation coefficients $(H)$ extracted for the less affected side of iSCl patients using four synergies. For the synergy vectors (W1-W4), each gray bar represents each iSCl patient, and the reference from the control group is represented in black. In relation to the activation coefficients $(\mathrm{H} 1-\mathrm{H} 4)$, gray lines represent the results of each iSCl patient and black lines represent the reference from the control group.

Table 5 Pearson correlations between the number of synergies, $\operatorname{VAF}_{\text {total }}$, vectors $\left(W \cdot W_{0}\right)$ and activation coefficients $\left(H \cdot H_{0}\right)$ and functional assessment scales, using four synergies to reconstruct the EMG envelopes of the less affected side (iSCl) and the most affected side (iSCla) of iSCl patients

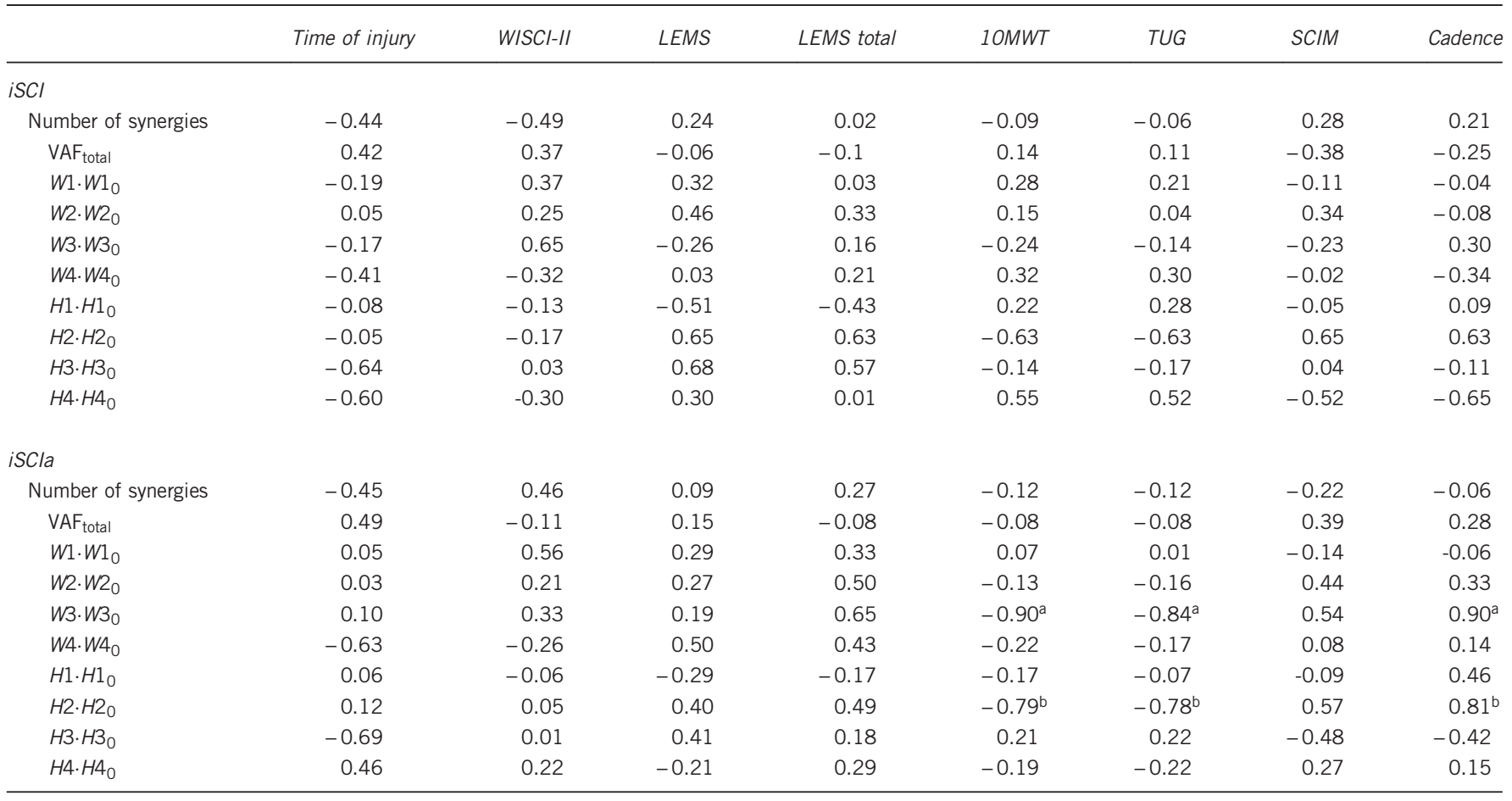

aCorrelation is significant at the 0.01 level.

${ }^{b}$ Correlation is significant at the 0.05 level. 

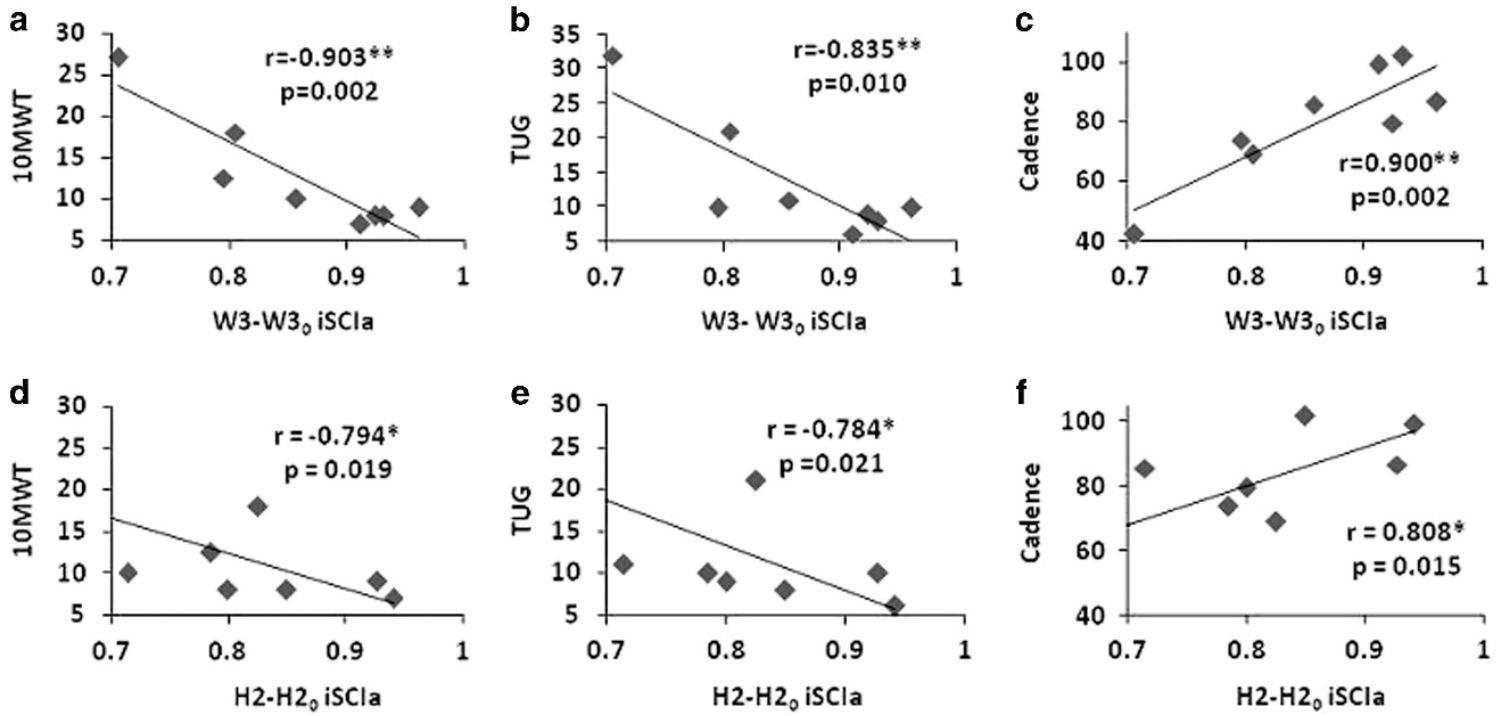

Figure 4 Correlation between functional gait assessment in the most affected side of iSCl and $W \cdot W_{0}$ and $H \cdot H_{0} \cdot{ }^{*}$ Correlation is significant at the 0.05 level (two-tailed). ${ }^{* *}$ Correlation is significant at the 0.01 level (two-tailed).

for the generation of the push-off before the swing phase. In the case of some iSCI patients, this synergy also contributed to the activation of other muscles (Figure 3), including the coactivation of TA (antagonists of Gas). For those cases, patients might have needed more time to take off the foot from the ground, which explains the worst performance in walking performance tests. We hypothesize that those patients with impaired control of synergies 2 and 3 may face more difficulty to compensate for these impairments and thus present worse walking performance.

When analyzing the possible correlations between the healthy-like structure of motor control and the motor performance of iSCI patients, no significant correlations were found between the number of synergies calculated for each side of iSCI patients and functional assessment scales, in opposition to what has been reported for poststroke patients. ${ }^{23}$ A possible reason could be the difference in pathologies being studied and different functional assessment scales being used. These preliminary results suggest that the number of synergies alone cannot be used to predict motor performance in iSCI patients.

Several limitations of this study need to be discussed. First, the small sample size limits the ability to detect statistical significance. The incomplete SCI is a very heterogeneous injury, ${ }^{25}$ which contributed to a great variability in walking patterns across different subjects, some of them requiring different levels of support from walking devices, which in turn may interfere in the synergies extraction and with natural gait. ${ }^{27,29}$ Nevertheless, we could not find a better way to compare these two groups, as it was also reported elsewhere. ${ }^{7,22}$ Another possible limitation of the study is the inclusion of only seven representative muscles of each side. It is possible to find or identify additional synergies if the repertoire of analyzed muscles is larger ${ }^{20}$ or the chosen muscles are different. ${ }^{37}$ Nevertheless, considering that we measured both sides, and included the markers for 3D gait analysis, we consider that we reached a good compromise between measurement completeness and minimal experimental burden, with the latter being a critical factor when analyzing SCI patients. ${ }^{16,18,23}$

The motor performance after a spinal cord injury evolves from the acute phase to the chronic period, and rehabilitation programs take advantage of the residual function both above and below the injury level. The main clinical potential of muscle synergies analysis is to provide a simplified view on specific neuro-mechanical mechanisms behind these residual motor functions, which are not visible under traditional clinical approaches. For instance, we observed that two biomechanical gait functions are mainly altered in iSCI population: one related to body weight support and the other related to the push-off. We showed that these alterations are reflected in two synergistic variables, specifically $H 2$ and $W 3$. Therefore, the analysis of synergy can provide the clinician with a tool to identify which specific mechanisms are more altered in a patient, and therefore to check and assess the evolution of these throughout the rehabilitation program. Furthermore, we observed that the impairment affects lower limb functions asymmetrically, and therefore support the importance of a bilateral analysis of muscle synergies, in order to provide additional information on asymmetric function that clinical tests are not able to grasp.

\section{CONCLUSION}

We showed how the analysis of muscle synergies can provide a more fine-grained view on the specific neuro-mechanical mechanisms behind residual motor functions, and therefore help the clinician to check their evolution throughout the recovery process and tailor the rehabilitation paradigm in a specific direction.

Our results showed that the similarity of specific synergies of the most affected side with the healthy reference presented correlations with clinical and functional walking scales. Specifically, patients presenting a synergistic control significantly different from the healthy reference also needed more time to execute these tests. We also demonstrated that asymmetry between both lower limbs is reflected in the structure of muscle synergies, and particularly in the $\mathrm{VAF}_{\text {total }}$ parameter. All these findings support the hypothesis that muscle synergy analysis is a valuable complementary outcome measure in addition to current clinical scales and metrics, and might be explored as a new neurophysiological metric to detect differences between the two sides in patients with iSCI.

To better quantify the cause-effect relationship between neuromuscular and functional behavior, future works should investigate the correlations between the structure of muscle synergies and jointspecific biomechanical data and other neurophysiologic measures of the spinal tracts, such as the ASIA motor and sensory index, or MRIbased measures of lesion extension. 


\section{DATA ARCHIVING}

There were no data to deposit.

\section{CONFLICT OF INTEREST}

The authors declare no conflict of interest.

\section{ACKNOWLEDGEMENTS}

This work is part of the HYPER project 'Hybrid Neuroprosthetic and Neurorobotic Devices for Functional Compensation and Rehabilitation of Motor Disorders' (ref. CSD2009-00067) funded by CONSOLIDER-INGENIO 2010, and the project ASSOCIATE 'A comprehensive and wearable robotics based approach to the rehabilitation and assistance to people with stroke and spinal cord injury' (ref. DPI2014-58431-C4-1-R), both funded by the Spanish Ministry for Science and Innovation. This research has been also supported by the European Commission 7th Framework Program as part of the project BioMot (FP7-ICT-2013-10, grant agreement no. 611695).

1 Wirz M, Zörner B, Rupp R, Dietz V. Outcome after incomplete spinal cord injury: central cord versus Brown-Sequard syndrome. Spinal Cord 2010; 48: 407-414.

2 Chang Ha, Chuang TY, Lee SJ, Liao SF, Lee HC, Shih YH et al. Temporal differences in relative phasing of gait initiation and first step length in patients with cervical and lumbosacral spinal cord injuries. Spinal Cord 2004; 42: 281-289.

3 Benito-Penalva J, Edwards DJ, Opisso E, Cortes M, Lopez-Blazquez R, Murillo N et al. Gait training in human spinal cord injury using electromechanical systems: effect of device type and patient characteristics. Arch Phys Med Rehabil 2012; 93: 404-412.

4 Wang P, Low KH, McGregor AH, Tow A. Detection of abnormal muscle activations during walking following spinal cord injury (SCI). Res Dev Disabil 2013; 34: 1226-1235.

5 Lacquaniti F, Ivanenko YP, D'Avella A, Zelik KE, Zago M. Evolutionary and developmental modules. Front Comput Neurosci 2013; 7: 61.

6 Gil-Agudo A, Pérez-Nombela S, Forner-Cordero A, Pérez-Rizo E, Crespo-Ruiz B, del Ama-Espinosa A. Gait kinematic analysis in patients with a mild form of central cord syndrome. J Neuroeng Rehabil 2011; 8: 7.

7 Gil-Agudo A, Solís-Mozos M, del-Ama AJ, Crespo-Ruiz B, de la Peña-González Al, PérezNombela S. Comparative ergonomic assessment of manual wheelchairs by paraplegic users. Disabil Rehabil Assist Technol 2013; 8: 305-313.

8 Barroso FO, Torricelli D, Moreno JC, Taylor J, Gomez-Soriano J, Bravo-Esteban E et al. Shared muscle synergies in human walking and cycling. J Neurophysiol 2014; 112: 1984-1998.

9 Cheung VC, Turolla A, Agostini M, Silvoni S, Bennis C, Kasi P et al. Muscle synergy patterns as physiological markers of motor cortical damage. Proc Natl Acad Sci USA 2012; 109: 14652-14656.

10 Dominici N, Ivanenko YP, Cappellini G, D’Avella A, Mondì V, Cicchese M et al. Locomotor primitives in newborn babies and their development. Science 2011; 334: 997-999.

11 Gizzi L, Nielsen JF, Felici F, Moreno JC, Pons JL, Farina D. Motor modules in robotaided walking. I Neuroeng Rehabil 2012; 9: 76.

12 Ivanenko YP, Cappellini G, Dominici N, Poppele RE, Lacquaniti F. Coordination of locomotion with voluntary movements in humans. J Neurosci 2005; 25: 7238-7253.

13 Routson RL, Clark DJ, Bowden MG, Kautz SA. Neptune RR. The influence of locomotor rehabilitation on module quality and post-stroke hemiparetic walking performance. Gait Posture 2013; 38: 511-517.

14 Allen JL, Neptune RR. Three-dimensional modular control of human walking. J Biomech 2012; 45: 2157-2163.
15 Neptune RR, Clark DJ, Kautz SA. Modular control of human walking: a simulation study. J Biomech 2009; 42: 1282-1287.

16 Fox EJ, Tester NJ, Kautz SA, Howland DR, Clark DJ, Garvan C et al. Modular control of varied locomotor tasks in children with incomplete spinal cord injuries. J Neurophysiol 2013; 110: 1415-1425.

17 Lacquaniti F, Ivanenko YP, Zago M. Patterned control of human locomotion. J Physiol 2012; 590 (Pt 10): 2189-2199.

18 Clark DJ, Ting LH, Zajac FE, Neptune RR, Kautz SA. Merging of healthy motor modules predicts reduced locomotor performance and muscle coordination complexity poststroke. J Neurophysiol 2010; 103: 844-857.

19 Ivanenko YP, Grasso R, Zago M, Molinari M, Scivoletto G, Castellano V et al. Temporal components of the motor patterns expressed by the human spinal cord reflect foot kinematics. J Neurophysiol 2003; 90: 3555-3565.

20 Ivanenko YP, Poppele RE, Lacquaniti F. Five basic muscle activation patterns account for muscle activity during human locomotion. J Physiol 2004; 556 (Pt 1): 267-282.

21 Cappellini G, Ivanenko YP, Poppele RE, Lacquaniti F. Motor patterns in human walking and running. J Neurophysiol 2006; 95: 3426-3437.

22 Gizzi L, Nielsen JF, Felici F, Ivanenko YP, Farina D. Impulses of activation but not motor modules are preserved in the locomotion of subacute stroke patients. I Neurophysiol 2011; 106: 202-210.

23 Bowden MG, Clark DJ, Kautz SA. Evaluation of abnormal synergy patterns poststroke: relationship of the Fugl-Meyer assessment to hemiparetic locomotion. Neurorehabil Neural Repair 2010; 24: 328-337.

24 Rodriguez KL, Roemmich RT, Cam B, Fregly BJ, Hass CJ. Persons with Parkinson's disease exhibit decreased neuromuscular complexity during gait. Clin Neurophysiol 2013; 124: 1390-1397.

25 Ting LH, Chiel HJ, Trumbower RD, Allen JL, McKay JL, Hackney ME et al. Neuromechanical principles underlying movement modularity and their implications for rehabilitation. Neuron 2015; 86: 38-54.

26 Grasso R, Ivanenko YP, Zago M, Molinari M, Scivoletto G, Castellano V et al. Distributed plasticity of locomotor pattern generators in spinal cord injured patients. Brain 2004; 127 (Pt 5): 1019-1034.

27 Ivanenko YP, Poppele RE, Lacquaniti F. Distributed neural networks for controlling human locomotion: lessons from normal and SCl subjects. Brain Res Bull 2009; 78: 13-21.

28 Lee SJ, Hidler J. Biomechanics of overground vs. treadmill walking in healthy individuals. J Appl Physiol 2008; 104: 747-755.

29 Hayes HB, Chvatal SA, French MA, Ting LH, Trumbower RD. Neuromuscular constraints on muscle coordination during overground walking in persons with chronic incomplete spinal cord injury. Clin Neurophysiol 2014; 125: 2024-2035.

30 Kapadia N, Masani K, Catharine Craven B, Giangregorio LM, Hitzig SL, Richards K et al. A randomized trial of functional electrical stimulation for walking in incomplete spinal cord injury: effects on walking competency. J Spinal Cord Med 2014; 37: 511-524.

31 Kadaba MP, Ramakrishnan HK, Wootten ME, Gainey J, Gorton G, Cochran GV. Repeatability of kinematic, kinetic, and electromyographic data in normal adult gait. J Orthop Res 1989; 7: 849-860.

32 Ochi F, Esquenazi A, Hirai B, Talaty M. Temporal-spatial feature of gait after traumatic brain injury. J Head Trauma Rehabil 1999; 14: 105-115.

33 Cram JR. Electrodes placements. In: Criswell E (ed.). Cram's Introduction to Surface Electromyography, 2nd edn. Jones \& Bartlett Publishers: Boston, MA, USA, 2011, pp 257-383.

34 Lee DD, Seung HS. Algorithms for non-negative matrix factorization. Adv Neural Inf Process Syst 2001; 1: 556-562.

35 Routson RL, Kautz SA, Neptune RR. Modular organization across changing task demands in healthy and poststroke gait. Physiol Rep 2014; 2: e12055.

36 Van Middendorp JJ, Pouw MH, Hayes KC, Williams R, Chhabra HS, Putz C et al. Diagnostic criteria of traumatic central cord syndrome. Part 2: a questionnaire survey among spine specialists. Spinal Cord 2010; 48: 657-663.

37 Steele KM, Tresch MC, Perreault EJ. The number and choice of muscles impact the results of muscle synergy analyses. Front Comput Neurosci 2013; 7: 105. 